\title{
Kaffee bei Herzschwäche? Kein Problem!
}

\begin{abstract}
Muss herzinsuffizienten Patienten wegen der Gefahr von Herzrhythmusstörungen und plötzlichem Herztod geraten werden, den Kaffeekonsum zu begrenzen? Eine experimentelle Studie mit hoch dosierter Koffeingabe spricht dagegen.
\end{abstract}

Kaffee gilt als ein Genussmittel, das bei übermäßigem Konsum Herzrhythmusstörungen verursachen kann. Viele Kardiologen empfehlen ihren ohnehin rhythmologisch gefährdeten Patienten mit höhergradiger Herzinsuffizienz deswegen, beim Kaffeekonsum zurückhaltend zu sein.

Diese Empfehlung beruhe aber auf eher wenigen, noch dazu kontroversen Daten, betonen brasilianische Kardiologen um Dr. Luis E. Rohde vom Krankenhaus Hospital de Clinicas in Porto Alegre.

Die Rhythmusspezialisten haben deswegen eine randomisierte Studie aufgelegt, an der unter streng kontrollierten Bedingungen 51 Patienten mit moderater bis schwerer linksventrikulärer Dysfunktion teilnahmen. Die durchschnittliche Ejektionsfraktion (EF) war mit 29\% deutlich eingeschränkt. Zwei von drei Patienten waren ICD-Träger. Alle Patienten tranken innerhalb von fünf Stunden jeweils fünf Tassen desselben entkoffeinierten Kaffees. Bei der Hälfte der Patienten wurden pro Tasse 100 mg Koffein zugegeben, bei den anderen $\mathrm{Pa}$ tienten dieselbe Menge Laktosepulver.

\section{Herz schlägt vor sich hin, ob mit Kaffee oder ohne}

Die 100 mg Koffein entsprechen einer eher starken Tasse Kaffee oder einem doppelten Espresso. Insgesamt nahmen die Patienten in der Verumgruppe damit $500 \mathrm{mg}$ Koffein ein, tranken also quasi eine komplette Kanne starken Kaffee. Um die Datenqualität zu verbessern, wählten die Brasilianer ein Crossover-Design, bei dem das Studienprotokoll nach einer Auswaschphase von einer Woche mit umgekehrten Vorzeichen wiederholt wurde.

Ab Einnahme der ersten Tasse Kaffee wurde bei jedem Patienten im Mittel 6,7 Stunden lang kontinuierlich das EKG überwacht. Eine Stunde nach der fünften Tasse Kaffee erfolgte eine Belastungsuntersuchung. Vor und nach der ersten und letzten Kaffeetasse wurden außerdem die Koffeinspiegel im Plasma bestimmt.

\section{Keine Hinweise auf}

\section{Dosis-Wirkungs-Beziehung}

Die insuffizienten Herzen zeigten sich vom Koffein insgesamt relativ unbeeindruckt. Statistisch signifikante Unterschiede beim Herzrhythmus zwischen den Konsumenten von Koffein und Laktosepulver fanden sich nicht. Ventrikuläre Extrasystolen traten in der Koffeingruppe $185 \mathrm{Mal}$ und in der Kontrollgruppe $239 \mathrm{Mal}$ auf, supraventrikuläre jeweils sechsmal. Auch bei Doppelschlägen, nicht anhaltenden Tachykardien und Bigeminus-Zyklen gab es keine signifikanten Unterschiede, genauso wenig in einer ganzen Reihe von Parametern, die unter Belastung erhoben wurden.

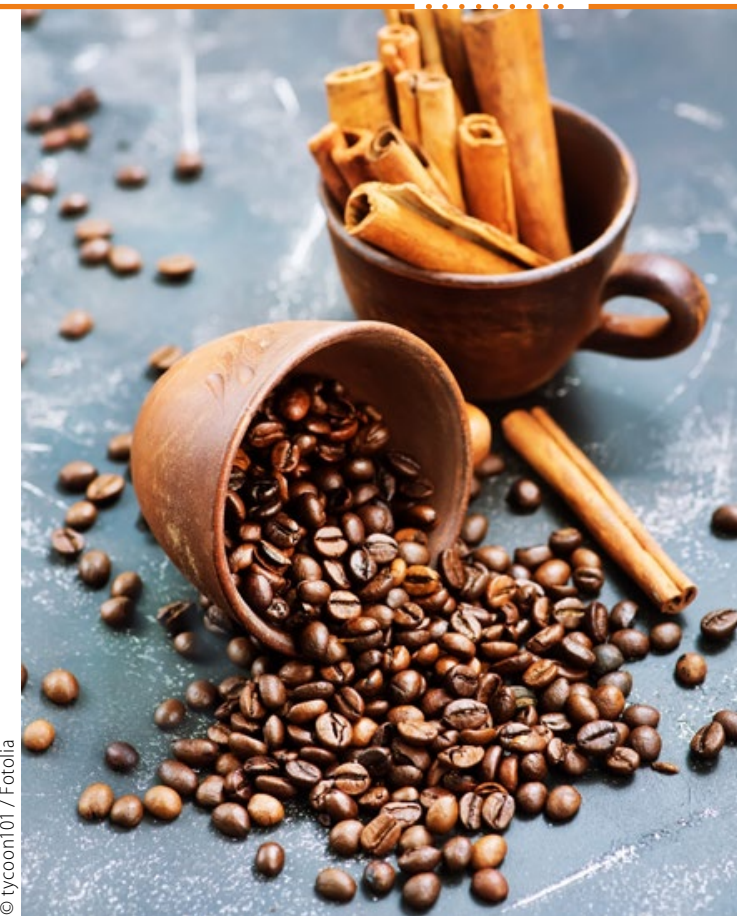

Lediglich der maximale Blutdruck unter Belastung war bei den Koffeinpatienten signifikant höher. Hinweise auf eine wie auch immer geartete Dosis-Wirkungs-Beziehung, festgemacht an den individuellen Plasmaspiegeln, fanden sich ebenfalls nicht.

Zuchinali P et al. Short-term Effects of High Dose Caffeine on Cardiac Arrhythmias in Patients With Heart Failure. JAMA Intern Med 2016. doi: 10.1001/jamainternmed.2016.6374

\section{Zusatztherapie bei Rückenschmerzen}

\section{Placebo wirkt auch bei eingeweihten Patienten}

\section{Placeboeffekte bei der Behandlung von Rückenschmerzen sind nicht davon abhängig, ob die Patienten über die Art der Therapie getäuscht werden oder nicht.}

In einer Studie wurden 83 Erwachsene mit chronischen Rückenschmerzen untersucht. 42 sollten drei Wochen lang ihre bisherige Therapie fortsetzen. 41 waren angehalten, zusätzlich zweimal täglich eine Placebopille einzunehmen. Zuvor wurden die Patienten darüber aufgeklärt, dass die Tabletten keine aktive Substanz enthalten, dass der
Körper aber auf Placebopillen automatisch reagieren könne, ähnlich wie der Pavlovsche Hund auf den Klingelton.

Der Schmerz, erfasst in einer Skala von 0 bis 10, setzte sich aus den Mittelwerten der minimalen, maximalen und üblichen Schmerzintensität zusammen. Der übliche sowie der maximale Schmerz besserten sich in der Placebogruppe jeweils signifikant um 30\%. In der Vergleichsgruppe gingen diese Werte nur um 9 bzw. 16\% zurück. (bs)

Carvalho Cet al. Pain 2016; online 13. Oktober; doi: 10.1097/j.pain.0000000000000700 